\title{
Envelhecimento acelerado em sementes de alface
}

\author{
Accelerated aging in lettuce seeds
}

\author{
Rafael Marani Barbosa ${ }^{\mathrm{I}}$ Denis Santiago da Costa ${ }^{\mathrm{II}}$ Marco Eustáquio de SáIII
}

\section{- NOTA -}

\section{RESUMO}

\begin{abstract}
Objetivou-se adequar a metodologia do teste de envelhecimento acelerado para avaliação do potencial fisiológico de sementes de quatro lotes dos genótipos de alface 'Babá de verão', 'Tainá'e 'Vera', aplicando-se o método tradicional e com o uso de solução saturada de $\mathrm{NaCl}$. Para tal, as sementes foram submetidas à determinação do teor de água, testes de germinação e vigor (primeira contagem, condutividade elétrica, emergência em bandeja, índice de velocidade de emergência) incluindo o teste de envelhecimento acelerado, conduzido nos períodos de 24,48 , e 72 horas, a $41{ }^{\circ} \mathrm{C}$ com e sem solução saturada de $\mathrm{NaCl}$. O experimento foi conduzido em delineamento inteiramente casualizado com quatro repetições. Os procedimentos tradicional e com o uso da solução saturada de $\mathrm{NaCl}$ por 72 horas foram sensíveis em estratificar os lotes em níveis de vigor, além de estarem de acordo com os demais testes utilizados. Portanto, o envelhecimento acelerado de sementes de alface deve ser feito utilizando o período de 72 horas, podendo ser realizado tanto pelo método tradicional quanto pelo método da solução saturada de $\mathrm{NaCl}$.
\end{abstract}

Palavras-chave: Lactuca sativa L., genótipos, vigor, solução saturada de $\mathrm{NaCl}$.

\section{ABSTRACT}

The objective was to adapt the methodology of the accelerated aging test to evaluate the physiological potential of seeds of four lots of lettuce genotypes 'Babá de verão', 'Tainá' and 'Vera' applying the traditional method and using a saturated $\mathrm{NaCl}$ solution. So, the seeds were subjected to determination of water content, seed germination and vigor (first count, electrical conductivity, speed emergency index, emergency tray) including the accelerated aging test conducted in periods of $24,48,72$ hours at $41^{\circ} \mathrm{C}$ with and without saturated solution of $\mathrm{NaCl}$. The experiment was conducted in a randomized design with four replications. The accelerated aging traditional and saturated solution of $\mathrm{NaCl}$ by $72 \mathrm{~h}$ was more sensitive to stratify the lots in vigor levels. Therefore, accelerated aging of seeds of lettuce should be made using the period of $72 \mathrm{~h}$ and can be accomplished either by the traditional method and by the saturated $\mathrm{NaCl}$ solution method.

Key words: Lactuca sativa L., genotype, vigor, saturated $\mathrm{NaCl}$ solution.

O teste de envelhecimento acelerado é considerado um dos mais sensíveis e eficientes para avaliação do vigor de sementes de diversas espécies (MARCOS FILHO, 2005). Entretanto, para sementes de hortaliças, o teste de envelhecimento acelerado pode ter certas limitações, devido ao tamanho pequeno e à grande variação no teor de água após o envelhecimento, resultando em deterioração acentuada e drástica redução da germinação (PANOBIANCO \& MARCOS FILHO, 2001). Assim, procedimentos com o uso de solução saturada de $\mathrm{NaCl}$ permitem a obtenção de umidades relativas inferiores às verificadas no

'Departamento de Produção Vegetal, Faculdade de Ciências Agrárias e Veterinárias (FCAV), Universidade Estadual Paulista "Júlio de Mesquita Filho" (UNESP), 14883-900, Jaboticabal, SP, Brasil. Email: rmarani@gmail.com. Autor para correspondência. "Departamento de Produção Vegetal, Escola Superior de Agricultura Luiz de Queiroz (ESALQ), Universidade de São Paulo (USP), Piracicaba, SP, Brasil.

"I'Departamento de Fitotecnia, Tecnologia de Alimentos e Socioeconomia, Faculdade de Engenharia, UNESP, Ilha Solteira, SP, Brasil. 
envelhecimento acelerado convencional (JIANHUA \& McDONALD, 1997).

No caso de sementes de alface, o período, a temperatura e o tipo de solução não estão bem esclarecidos pela literatura. FRANZIN et al. (2004), avaliando metodologias para estimar o potencial fisiológico de sementes de alface, observaram que o envelhecimento acelerado tradicional conduzido a $41^{\circ} \mathrm{C}$ por $72 \mathrm{~h}$ é um dos testes mais indicado para estimar o potencial fisiológico. Por outro lado, NASCIMENTO \& PEREIRA (2007) verificaram que o envelhecimento acelerado tradicional conduzido a $41^{\circ} \mathrm{C}$ por $48 \mathrm{~h}$ pode ser utilizado como parâmetro para escolha dos lotes.

Diante do exposto, objetivou-se adequar a metodologia do teste de envelhecimento acelerado para avaliação do potencial fisiológico de sementes de alface aplicando-se o método tradicional e com o uso de solução saturada de $\mathrm{NaCl}$.

O experimento foi desenvolvido no Laboratório de Análises de Sementes, da Faculdade de Engenharia de Ilha Solteira, UNESP - Campus de Ilha Solteira/SP, com sementes de quatro lotes de três cultivares de alface: 'Babá de verão', 'Tainá' e 'Vera'. As sementes foram submetidas a determinações de teor de água e a testes de germinação e vigor a seguir descritos: Teor de água: determinado pelo método da estufa a $105 \pm 3^{\circ} \mathrm{C}$, durante 24 horas, com duas amostras com 0,5g de sementes para cada lote (BRASIL, 2009), antes e após os períodos de envelhecimento. Germinação: quatro repetições de 50 sementes foram semeadas em caixas de plástico transparente $(11 \times 11 \times 3,5 \mathrm{~cm})$, sobre papel de filtro umedecido com água destilada na proporção de 2,5 vezes o seu peso seco. As caixas foram mantidas em câmara de germinação a $25^{\circ} \mathrm{C}$ constantes, com fotoperíodo de oito horas. As avaliações foram feitas aos quatro e sete dias, e os resultados expressos em porcentagem de plântulas normais (BRASIL, 2009). Primeira contagem: constou da avaliação da porcentagem de plântulas normais no quarto dia após a instalação do teste de germinação. Condutividade elétrica: quatro repetições de 50 sementes foram pesadas com precisão de $0,01 \mathrm{~g}$, imersas em $75 \mathrm{~mL}$ de água desionizada, em copos de plástico $(200 \mathrm{~mL})$ e mantidas a $25^{\circ} \mathrm{C}$, durante 24 horas. Após esse período, a leitura foi realizada em aparelho condutivímetro Digimed CD-21 e os resultados expressos em $\mu \mathrm{S} \mathrm{cm}^{-1} \mathrm{~g}^{-1}$ (MARCOS FILHO \& VIEIRA, 2009). Emergência de plântulas em bandeja: foram semeadas a $2 \mathrm{~cm}$ de profundidade, quatro repetições de 50 sementes, em células de bandeja de isopor contendo o substrato Plantmax ${ }^{\circledast}$ Hortaliça. A contagem das plântulas foi realizada no $12^{\circ}$ dia após o início do teste, considerando as plântulas normais aquelas cujas folhas mediam $2 \mathrm{~cm}$ acima da superfície do substrato. Avaliou-se a velocidade de emergência de plântulas, mediante a contagem diária de plântulas emergidas, e a porcentagem de emergência de plântulas aos 12 dias após a semeadura. O cálculo do índice de velocidade de emergência (IVE) foi realizado de acordo com MAGUIRE (1962). Envelhecimento acelerado tradicional (EAT): realizado em caixas de plástico transparente $(11 \times 11 \times 3,5 \mathrm{~cm})$, contendo em seu interior tela metálica acoplada e uma tela de nylon sobreposta para dar suporte às sementes. Dentro das caixas, foram colocados $40 \mathrm{~mL}$ de água destilada e, após tampadas, mantidas no interior de câmaras de envelhecimento a $41^{\circ} \mathrm{C}$, durante 24,48 e 72 horas. Decorrido os períodos de envelhecimento, as sementes foram colocadas para germinar seguindo metodologia descrita para a germinação e a avaliação realizada no quarto dia após a semeadura. Envelhecimento acelerado com solução saturada de $\mathrm{NaCl}$ (EASS): conduzido de forma semelhante à descrita para o procedimento tradicional, substituindo-se os $40 \mathrm{~mL}$ de água destilada por igual quantidade de solução saturada de $\mathrm{NaCl}(40 \mathrm{~g}$ em $100 \mathrm{mLde}$ água) (JIANHUA \& McDONALD, 1997).

O delineamento utilizado foi inteiramente casualizado com quatro repetições. Os dados foram submetidos à análise de variância e as médias comparadas peloteste de Tukey $(\mathrm{P} \leq 0,05)$, separadamente para cada cultivar, sendo que os dados que não obtiveram a distribuição normal foram previamente transformados em arc sen $(\mathrm{x} / 100)^{1 / 2}$ (BANZATO \& KRONKA, 2006).

Pequenas variações entre os teores de água inicial dos lotes das sementes de cada cultivar de alface foram encontrados: 0,7; 0,8 e 0,9\% para as cultivares 'Babá de verão', 'Tainá' e 'Vera', respectivamente. A exposição das sementes ao envelhecimento acelerado ocasionou aumento no teor de água (Tabela 1). A velocidade de absorção e a quantidade de água absorvida pelas sementes foram maiores para aquelas envelhecidas sob solução tradicional, de modo que esse aumento variou em função do período de envelhecimento.

Pelos resultados do teste de germinação de sementes das cultivares 'Babá de verão', 'Tainá' e 'Vera' (Tabela 2), constataram-se similaridades entre os lotes, no entanto os demais testes aplicados identificaram diferenças de vigor entre eles. Pelos resultados da primeira contagem de germinação, índice de velocidade de emergência e condutividade elétrica, constatou-se desempenho inferior das sementes dos lotes C ('Babá de verão'), H ('Tainá') e J ('Vera'), sendo que, para os demais lotes, de alto vigor, o comportamento foi semelhante. Por outro lado, a emergência de plântulas 
Tabela 1 - Teor de água (\%) de sementes de alface cv. 'Babá de verão', 'Tainá' e 'Vera' antes e após o envelhecimento acelerado tradicional (EAT) e com solução saturada de $\mathrm{NaCl}$ (EASS) sob diferentes períodos. Ilha Solteira, 2009.

\begin{tabular}{|c|c|c|c|c|c|c|c|}
\hline \multirow{2}{*}{ Lotes } & \multirow{2}{*}{ Inicial } & \multicolumn{2}{|c|}{--24h--------------- } & \multicolumn{2}{|c|}{ 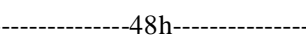 } & \multicolumn{2}{|c|}{----------72h----------- } \\
\hline & & EAT & EASS & EAT & EASS & EAT & EASS \\
\hline A & 6,1 & 16,2 & 7,1 & 23,4 & 9,7 & 31,7 & 10,2 \\
\hline B & 5,9 & 17,9 & 8,6 & 21,9 & 11,1 & 32,4 & 12,6 \\
\hline $\mathrm{C}$ & 5,4 & 18,7 & 7,4 & 22,3 & 10,6 & 31,9 & 10,9 \\
\hline $\mathrm{D}$ & 5,5 & 16,4 & 7,9 & 22,9 & 9,8 & 33,0 & 12,3 \\
\hline $\mathrm{E}$ & 6,3 & 17,1 & 8,3 & 21,5 & 9,9 & 32,9 & 11,8 \\
\hline $\mathrm{F}$ & 5,8 & 18,2 & 8,7 & 20,7 & 10,5 & 33,5 & 12,4 \\
\hline G & 6,0 & 17,9 & 7,9 & 24,2 & 9,4 & 31,3 & 12,6 \\
\hline $\mathrm{H}$ & 5,5 & 16,5 & 6,9 & 24,0 & 10,6 & 30,4 & 11,5 \\
\hline I & 5,7 & 18,8 & 7,8 & 21,0 & 10,3 & 33,1 & 12,2 \\
\hline $\mathrm{J}$ & 5,9 & 17,7 & 8,9 & 23,7 & 10,9 & 34,7 & 12,7 \\
\hline $\mathrm{K}$ & 6,1 & 16,1 & 8,8 & 22,4 & 11,4 & 31,9 & 11,6 \\
\hline $\mathrm{L}$ & 5,2 & 16,6 & 7,7 & 22,9 & 9,9 & 34,4 & 11,9 \\
\hline
\end{tabular}

Tabela 2 - Germinação - GE (\%) e vigor: primeira contagem da germinação - PC $(\%)$, índice de velocidade de germinação - IVE, condutividade elétrica - CE $(\mu \mathrm{S}$ $\mathrm{cm}^{-1} \mathrm{~g}^{-1}$ ) e emergência de plântulas - EM (\%) de diferentes lotes de sementes de alface cv. 'Babá de

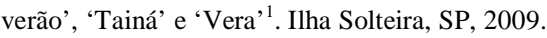

\begin{tabular}{|c|c|c|c|c|c|}
\hline Lotes & $\mathrm{GE}^{2}$ & $\mathrm{PC}^{2}$ & IVE & $\mathrm{CE}$ & $\mathrm{EM}^{2}$ \\
\hline \multicolumn{6}{|c|}{ 'Babá de verão' } \\
\hline A & $97 \mathrm{a}$ & $94 \mathrm{a}$ & $12,0 \mathrm{a}$ & $28 \mathrm{~b}$ & $94 \mathrm{a}$ \\
\hline B & $92 \mathrm{a}$ & $89 a$ & $11,3 \mathrm{a}$ & $32 \mathrm{~b}$ & $96 \mathrm{a}$ \\
\hline $\mathrm{C}$ & $92 \mathrm{a}$ & $80 \mathrm{~b}$ & $10,4 \mathrm{~b}$ & $59 a$ & $81 \mathrm{~b}$ \\
\hline $\mathrm{D}$ & $94 \mathrm{a}$ & $89 \mathrm{a}$ & $11,5 \mathrm{a}$ & $37 \mathrm{~b}$ & $92 \mathrm{a}$ \\
\hline \multicolumn{6}{|c|}{ ‘Tainá' } \\
\hline $\mathrm{E}$ & $99 a$ & $97 \mathrm{a}$ & $12,3 \mathrm{a}$ & $31 \mathrm{c}$ & $90 \mathrm{a}$ \\
\hline $\mathrm{F}$ & $95 \mathrm{a}$ & $89 a$ & $11,2 \mathrm{a}$ & $52 \mathrm{~b}$ & $81 \mathrm{a}$ \\
\hline G & $94 \mathrm{a}$ & $90 \mathrm{a}$ & $11,4 \mathrm{a}$ & $50 \mathrm{~b}$ & $84 \mathrm{a}$ \\
\hline $\mathrm{H}$ & $93 a$ & $77 \mathrm{~b}$ & $9,4 \mathrm{~b}$ & $69 a$ & $80 a$ \\
\hline \multicolumn{6}{|c|}{ 'Vera' } \\
\hline I & $97 \mathrm{a}$ & $94 \mathrm{a}$ & $12,0 \mathrm{a}$ & $52 \mathrm{~b}$ & $83 \mathrm{a}$ \\
\hline $\mathrm{J}$ & $93 a$ & $62 \mathrm{c}$ & $9,0 \mathrm{~b}$ & $69 a$ & $74 b$ \\
\hline $\mathrm{K}$ & $91 \mathrm{a}$ & $80 \mathrm{~b}$ & $12,8 \mathrm{a}$ & $44 \mathrm{~b}$ & $89 a$ \\
\hline $\mathrm{L}$ & $96 a$ & $88 \mathrm{~b}$ & $11,6 \mathrm{a}$ & $41 \mathrm{~b}$ & $90 \mathrm{a}$ \\
\hline DMS (Babá) & 6,4 & 6,7 & 1,3 & 20,4 & 10,9 \\
\hline DMS (Tainá) & 7,9 & 8,4 & 1,6 & 15,4 & 12,8 \\
\hline DMS (Vera) & 6,7 & 5,4 & 1,2 & 16,1 & 12,3 \\
\hline $\mathrm{CV}(\%)$ & 6,2 & 7,4 & 6,2 & 25,3 & 15,7 \\
\hline
\end{tabular}

${ }^{1}$ Médias seguidas de mesma letra, na coluna, não diferem entre si em nível de $5 \%$ de probabilidade pelo teste de Tukey. ${ }^{2}$ Dados originais, mas, para efeito de análise estatística, foram transformados em $\operatorname{arcsen}(\mathrm{x} / 100)^{1 / 2}$. identificou diferenças entre os lotes das cultivares 'Babá de verão' e 'Vera', com destaque para os lotes C e $\mathbf{J}$ com qualidade inferior, porém não distinguiu os lotes da cv. 'Tainá'.

Pelos resultados do teste de envelhecimento acelerado (Tabela 3), não foi observado haver possibilidade de classificação dos lotes de sementes de alface, pelos períodos de 24 e 48 horas, tanto o EAT quanto EASS, pois os resultados não foram concordantes com os demais testes.

Nos testes de EAT e EASS, conduzidos por 72 horas (Tabela 3) observou-se separação dos lotes quanto ao nível de vigor, dentre cada cultivar. Esses resultados estão em consonância com aqueles obtidos junto aos outros testes aplicados, exceto para a emergência de plântulas (Tabela 2). Dessa forma, o envelhecimento acelerado tradicional por 72 horas foi sensível o suficiente para identificar diferenças entre lotes. Esse resultado concorda com HAMPTON \& TEKRONY (1995), que apontam o envelhecimento acelerado com solução tradicional a $41^{\circ} \mathrm{C}$ por período de 72 horas como ideal para estimar vigor em sementes de alface. Por outro lado, a combinação que proporcionou melhor separação dos lotes foi o envelhecimento acelerado das sementes por 72 horas a $41^{\circ} \mathrm{C}$ com solução saturada de $\mathrm{NaCl}$, demonstrando maior potencial para separação dos lotes em níveis de vigor.

$\mathrm{O}$ envelhecimento acelerado em sementes de alface deve ser realizado pelo período de 72 horas, podendo ser feito tanto pelo método tradicional, quanto 
Tabela 3 - Vigor de diferentes lotes de sementes de alface cv. 'Babá de verão', 'Tainá' e 'Vera' após o teste de envelhecimento acelerado tradicional (EAT) e com uso de solução saturada de $\mathrm{NaCl}$ (EASS), em três períodos de envelhecimento ${ }^{1,2}$. Ilha Solteira, SP, 2009.

\begin{tabular}{|c|c|c|c|c|c|c|}
\hline \multirow{2}{*}{ Lotes } & \multicolumn{3}{|c|}{-EAT- } & \multicolumn{3}{|c|}{----EASS-- } \\
\hline & $24 \mathrm{~h}$ & $48 \mathrm{~h}$ & $72 \mathrm{~h}$ & $24 \mathrm{~h}$ & $48 \mathrm{~h}$ & $72 \mathrm{~h}$ \\
\hline & & & & & & \\
\hline A & $92 \mathrm{a}$ & $73 \mathrm{a}$ & $24 \mathrm{a}$ & $92 \mathrm{a}$ & $91 \mathrm{a}$ & $29 \mathrm{~b}$ \\
\hline B & $91 \mathrm{a}$ & $71 \mathrm{a}$ & $19 \mathrm{a}$ & $92 \mathrm{a}$ & $87 \mathrm{a}$ & $35 \mathrm{a}$ \\
\hline $\mathrm{C}$ & $88 \mathrm{a}$ & $65 \mathrm{~b}$ & $16 \mathrm{~b}$ & $90 \mathrm{a}$ & $69 \mathrm{~b}$ & $25 \mathrm{c}$ \\
\hline $\mathrm{D}$ & $90 \mathrm{a}$ & $66 \mathrm{~b}$ & $22 \mathrm{a}$ & $88 \mathrm{a}$ & $75 \mathrm{ab}$ & $34 \mathrm{a}$ \\
\hline E & $91 \mathrm{a}$ & $70 \mathrm{a}$ & $19 \mathrm{a}$ & $90 \mathrm{a}$ & $82 \mathrm{a}$ & $28 \mathrm{a}$ \\
\hline $\mathrm{F}$ & $90 \mathrm{a}$ & $71 \mathrm{a}$ & $18 \mathrm{a}$ & $90 \mathrm{a}$ & $79 \mathrm{a}$ & $22 \mathrm{~b}$ \\
\hline $\mathrm{G}$ & $88 \mathrm{a}$ & $67 \mathrm{~b}$ & $21 \mathrm{a}$ & $88 \mathrm{a}$ & $82 \mathrm{a}$ & $21 \mathrm{~b}$ \\
\hline $\mathrm{H}$ & $88 \mathrm{a}$ & $62 \mathrm{c}$ & $10 \mathrm{~b}$ & 89 a & $76 \mathrm{a}$ & $16 \mathrm{c}$ \\
\hline I & $90 \mathrm{a}$ & $76 b$ & $21 \mathrm{a}$ & $94 \mathrm{a}$ & $78 \mathrm{a}$ & $29 \mathrm{~b}$ \\
\hline $\mathrm{J}$ & $89 a$ & $63 \mathrm{c}$ & $14 \mathrm{~b}$ & $92 \mathrm{a}$ & $72 \mathrm{a}$ & $23 \mathrm{c}$ \\
\hline K & $92 \mathrm{a}$ & $79 \mathrm{a}$ & $19 \mathrm{a}$ & $96 \mathrm{a}$ & $79 \mathrm{a}$ & $35 \mathrm{a}$ \\
\hline $\mathrm{L}$ & $90 \mathrm{a}$ & $80 \mathrm{a}$ & $22 \mathrm{a}$ & $94 \mathrm{a}$ & $80 \mathrm{a}$ & $29 \mathrm{~b}$ \\
\hline DMS ('Babá') & 8,9 & 4,7 & 3,8 & 6,9 & 17,2 & 3,9 \\
\hline DMS ('Tainá') & 5,3 & 2,8 & 3,5 & 5,8 & 11,9 & 4,8 \\
\hline DMS ('Vera') & 14,7 & 2,7 & 4,5 & 6,3 & 12,1 & 5,4 \\
\hline CV (\%) & 10,9 & 15,7 & 11,3 & 12,7 & 10,4 & 14,8 \\
\hline
\end{tabular}

${ }^{1}$ Médias seguidas de mesma letra, na coluna, não diferem entre si em nível de 5\% de probabilidade pelo teste de Tukey. ${ }^{2}$ Dados originais, mas, para efeito de análise estatística, foram transformados em $\operatorname{arcsen}(\mathrm{x} / 100)^{1 / 2}$.

pelo uso de solução saturada de $\mathrm{NaCl}$, independentemente da cultivar estudada.

\section{REFERÊNCIAS}

BANZATO, D.A.; KRONKA, S.N. Experimentação agrícola 4.ed. Jaboticabal: FUNEP, 2006. 237p.

BRASIL. Ministério da Agricultura, Pecuária e Abastecimento. Regras para análise de sementes. Secretaria de Defesa Agropecuária. Brasília: MAPA/ACS, 2009. 395p.

FRANZIN, S.M. et al. Métodos para avaliação do potencial fisiológico de sementes de alface. Revista Brasileira de Sementes, Pelotas, v.26, n.2, p.63-69, 2004. Disponível em: $\langle$ http://www.scielo.br/pdf/rbs/v26n2/24490.pdf >. Acesso em: 01 abr. 2011. doi: 10.1590/S0101-31222004000200009.

HAMPTON, J.G.; TEKRONY, D.M. (ed.). Handbook of vigour test methods. 3.ed. Zurich: International Seed Testing Association, 1995. $117 \mathrm{p}$.

JIANHUA, Z.; McDONALD, M.B. The saturated salt accelerated aging test for small-seeded crops. Seed Science and Technology, Zürich, v.25, n.1, p.123-131, 1997.
MAGUIRE, J.D. Speed of germination and in selection and evaluation for seedling emergence and vigor. Crop Science, Madison, v.2, n.2, p.176-177, 1962.

MARCOS FILHO, J.; VIEIRA, R.D. Seed vigor tests: procedures conductivity tests. In: BAALBAKI, R. et al. (Orgs). Seed vigor tests handbook. Ithaca, NY, USA: AOSA, 2009. p.186-200.

MARCOS FILHO, J. Fisiologia de sementes de plantas cultivadas. Piracicaba: FEALQ, 2005. 495p.

NASCIMENTO, W.M.; PEREIRA, R.S. Testes para avaliação do potencial fisiológico de sementes de alface e sua relação com a germinação sob temperaturas adversas. Revista Brasileira de Sementes, Pelotas, v.29, n.3, p.175-179, 2007. Disponível em: <http://www.scielo.br/pdf/rbs/v29n3/a21v29n3.pdf>. Acesso em: 15 ago. 2010. doi: 10.1590/S010131222007000300021 .

PANOBIANCO, M.; MARCOS FILHO, J. Envelhecimento acelerado e deterioração controlada em sementes de tomate. Scientia Agricola, Piracicaba, v.58, n.3, p.525-531, 2001. Disponível em: <http://www.scielo.br/pdf/sa/v58n3/a14v58n3.pdf. Acesso em: 19 set. 2010. doi: 10.1590/S0103-90162001000300014. 